\title{
Multiobjective optimization design of an rf gun based electron diffraction beam line
}

\author{
Colwyn Gulliford, Adam Bartnik, and Ivan Bazarov \\ CLASSE, Cornell University, 161 Synchrotron Drive, Ithaca, New York 14853-8001, USA \\ Jared Maxson \\ Department of Physics and Astronomy, University California,
}

Los Angeles, Los Angeles, California 90095, USA

(Received 14 October 2016; published 2 March 2017)

\begin{abstract}
Multiobjective genetic algorithm optimizations of a single-shot ultrafast electron diffraction beam line comprised of a $100 \mathrm{MV} / \mathrm{m}$ 1.6-cell normal conducting rf (NCRF) gun, as well as a nine-cell $2 \pi / 3$ bunching cavity placed between two solenoids, have been performed. These include optimization of the normalized transverse emittance as a function of bunch charge, as well as optimization of the transverse coherence length as a function of the rms bunch length of the beam at the sample location for a fixed charge of $10^{6}$ electrons. Analysis of the resulting solutions is discussed in terms of the relevant scaling laws, and a detailed description of one of the resulting solutions from the coherence length optimizations is given. For a charge of $10^{6}$ electrons and final beam sizes of $\sigma_{x} \geq 25 \mu \mathrm{m}$ and $\sigma_{t} \approx 5 \mathrm{fs}$, we found a relative coherence length of $L_{c, x} / \sigma_{x} \approx 0.07$ using direct optimization of the coherence length. Additionally, based on optimizations of the emittance as a function of final bunch length, we estimate the relative coherence length for bunch lengths of 30 and $100 \mathrm{fs}$ to be roughly 0.1 and $0.2 \mathrm{~nm} / \mu \mathrm{m}$, respectively. Finally, using the scaling of the optimal emittance with bunch charge, for a charge of $10^{5}$ electrons, we estimate relative coherence lengths of $0.3,0.5$, and $0.92 \mathrm{~nm} / \mu \mathrm{m}$ for final bunch lengths of 5, 30 and $100 \mathrm{fs}$, respectively.
\end{abstract}

DOI: 10.1103/PhysRevAccelBeams.20.033401

\section{INTRODUCTION}

The desire for single-shot ultrafast electron diffraction (UED) beam lines with sufficient resolution $\left(\sigma_{t} \lesssim 30 \mathrm{fs}\right.$, $q \sim 10^{6}$ electrons) for use with biological samples, such as proteins, continues to push the development of both photocathode and cold atom electron sources [1-17]. For photoemission UED sources such as DC and normal conducting rf guns (NCRF) [18], the charge and beam sizes at the cathode require optimal emittance preservation of a space charged dominated beam. Building on the successful application of multiobjective genetic algorithm (MOGA) optimized simulations of space charge dominated beams used in the design and operation of the Cornell photoinjector [19-21], as well as the optimization of a cryocooled DC gun UED setup [22], we apply the same techniques to a $100 \mathrm{MV} / \mathrm{m}$ 1.6-cell NCRF gun followed by a nine-cell, $2 \pi / 3$ buncher cavity. We use a mean transverse energy (MTE) of $35 \mathrm{meV}$ for the simulated photoelectrons, a value considered achievable through the use of multialkali photocathodes operated near threshold [23].

\footnotetext{
*cg248@cornell.edu
}

Published by the American Physical Society under the terms of the Creative Commons Attribution 4.0 International license. Further distribution of this work must maintain attribution to the author(s) and the published article's title, journal citation, and DOI.
The NCRF gun based UED setup in this work consists of a 1.6-cell $2.856 \mathrm{GHz}$ NCRF gun followed by a buncher cavity placed between two solenoid magnets [24-26]. For all simulations, the maximum allowable peak cathode field is set to $100 \mathrm{MV} / \mathrm{m}$ and the cathode MTE is fixed to $35 \mathrm{meV}$ for all simulations. For the buncher model, we used the dimensions of the first cell in the SLAC linac [27] (see Table I) repeated a total of 9 times, to make a nine-cell $2 \pi / 3$ traveling wave buncher cavity. The field maps were generated in Poisson SUPERFISH [28].

To model solenoids similar to those in $[24,25]$ we use an analytic form for the on-axis solenoid field from a sheet of current with radius $R$ and length $L$,

$$
B_{z}(z)=B_{0}\left(\frac{\Delta z_{+}}{\sqrt{\Delta z_{+}^{2}+R^{2}}}-\frac{\Delta z_{-}}{\sqrt{\Delta z_{-}^{2}+R^{2}}}\right),
$$

where $\Delta z_{ \pm}=z \pm L / 2$ and fit this model to solenoid field map data [22]. The fields used in space charge simulations are computed from the third order off-axis expansion of Eq. (1). Figure 1 shows the on axis field data for the beamline setup.

\section{RESULTS}

For this work, we make use of the same MOGA software used in previous work $[19,20,22]$ in combination with the 3D space charge code general particle tracer (GPT). In each 
TABLE I. Buncher cavity design parameters.

\begin{tabular}{lc}
\hline \hline Parameter & Value \\
\hline Period $d$ & $3.5 \mathrm{~cm}$ \\
Cell diameter $2 b$ & $8.34 \mathrm{~cm}$ \\
Disk hole diameter $2 a$ & $2.62 \mathrm{~cm}$ \\
Disk thickness $t$ & $0.58 \mathrm{~cm}$ \\
Disk edge radius $\rho$ & $0.31 \mathrm{~cm}$ \\
\hline \hline
\end{tabular}

optimization, the optimizer was allowed to vary the laser rms sizes, beam line element parameters and positions, as well as arbitrarily shape both the transverse and longitudinal laser distributions, based on the same methods described in $[22,29]$. The set of beam line parameters varied are shown in Table II. In all optimizations the population size was set to 50. The number of generations run for each optimization ranged between several hundred to a several thousand, depending on whether the optimization was starting from noise or being seeded from a previous solution. Progression of the front was monitored every 10 to 20 generations after an initial 200. When no discernible improvement of the front was found, the optimization was stopped.

\section{A. Optimal emittance}

Fundamentally, for a given bunch charge, the coherence length at the sample is limited by the cathode emittance produced in the charge saturation limit, as well as the ability to preserve this emittance while transporting and focusing the beam down to the required beam sizes at the sample [22]. For a given charge $q$ extracted in the charge saturation limit for an accelerating field $E_{0}$, the limiting emittance scales as

$$
\epsilon_{n, x, i} \propto \sqrt{\frac{\operatorname{MTE}}{m_{e} c^{2}}} \begin{cases}\left(\frac{q}{\varepsilon_{0} E_{0}}\right)^{1 / 2}, & A \gg 1 \text { (“pancake") } \\ \frac{1}{E_{0}}\left(\frac{q}{\sigma_{t, i}}\right)^{2 / 3}, & A \leq 1 \text { (“cigar”). }\end{cases}
$$

TABLE II. Beam line simulation parameters.

\begin{tabular}{lc}
\hline \hline Parameter & Range \\
\hline Laser size $\sigma_{t, i}$ & {$[0,20] \mathrm{ps}$} \\
Laser size $\sigma_{x, i}$ & {$[0,1] \mathrm{mm}$} \\
Cathode MTE & $35 \mathrm{meV}$ \\
Peak gun field & $100 \mathrm{MV} / \mathrm{m}$ \\
Gun phase & {$[0,360] \mathrm{deg}$} \\
Solenoid radius/length & $2.53 / 9.54 \mathrm{~cm}$ \\
Solenoid peak field(s) & {$[0,1.0] \mathrm{T}$} \\
Solenoid 1 position & {$[0.25,5.25] \mathrm{m}$} \\
Peak buncher power & {$[0,25] \mathrm{MW}$} \\
Buncher phase & {$[0,360] \mathrm{deg}$} \\
Buncher position & {$[0.4,10.4] \mathrm{m}$} \\
Solenoid 2 position & {$[0.55,15.55] \mathrm{m}$} \\
Sample position & {$[0.5520 .55] \mathrm{m}$} \\
\hline \hline
\end{tabular}

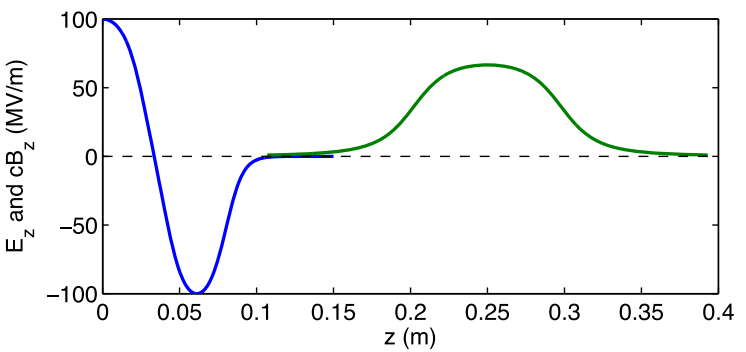

(a) Example of the on-axis accelerating (blue) and solenoid field profiles (green).

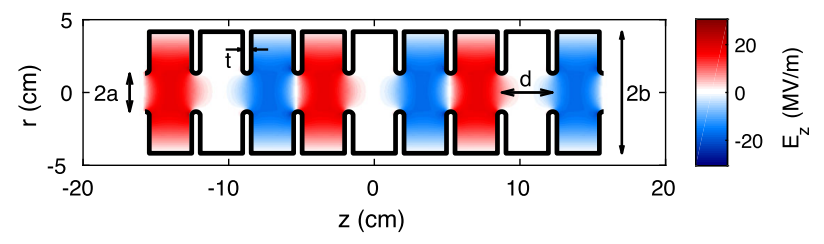

FIG. 1. Field description for the 1.6-cell gun and solenoid (a), as well as the buncher (b).

In this expression $\sigma_{t, i}$ is the rms laser pulse length, and $A=\sigma_{x, i} /\left(\frac{e E_{0}}{m_{e} c^{2}}\right)\left(c \sigma_{t, i}\right)^{2}$ is the initial beam aspect ratio. Note the limit $A \ll 1$ describes the short initial bunch regime.

To verify the validity of Eq. (2), we performed an initial round of emittance optimizations for a "long" final beam $\sigma_{t} \leq 500 \mathrm{fs}$ (the bunch length is also minimized later), while constraining the transverse spot size: $\sigma_{x} \leq 25 \mu \mathrm{m}$, and simultaneously maximizing the bunch charge. In these optimizations, we require that no particles are lost in beam transport. Figure 2 shows the emittance performance. The figure also shows the results of fitting the emittance data with both charge scaling laws found in Eq. (2) for beams extracted in the charge saturation limit, and suggests that the emittance at the sample scales as $q^{2 / 3}$. Noting that the optimizer chose an initial laser pulse length of roughly 2.4 ps for the charges shown, and computing the initial beam aspect ratio for all points on the front leads to an

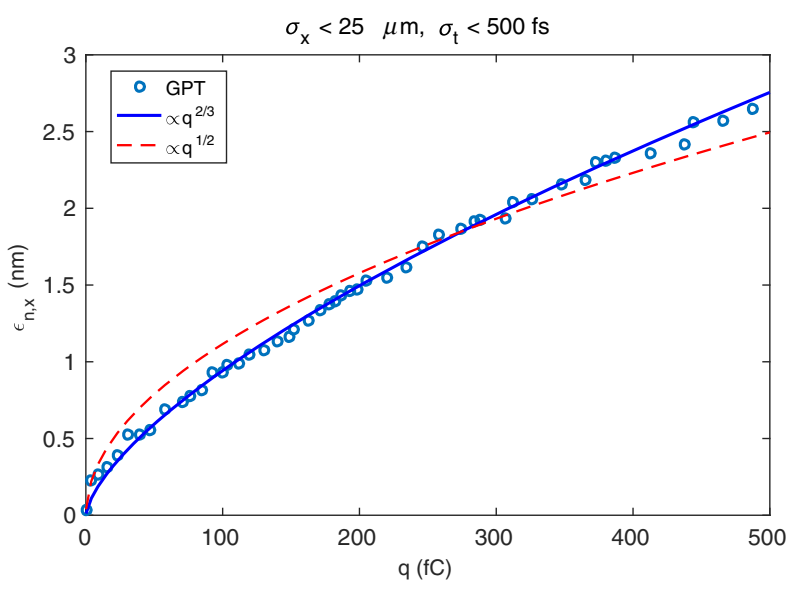

FIG. 2. Optimal emittance as a function of bunch charge at the sample for a final beam size of $\sigma_{x} \leq 25, \sigma \leq 500 \mathrm{fs}$. 


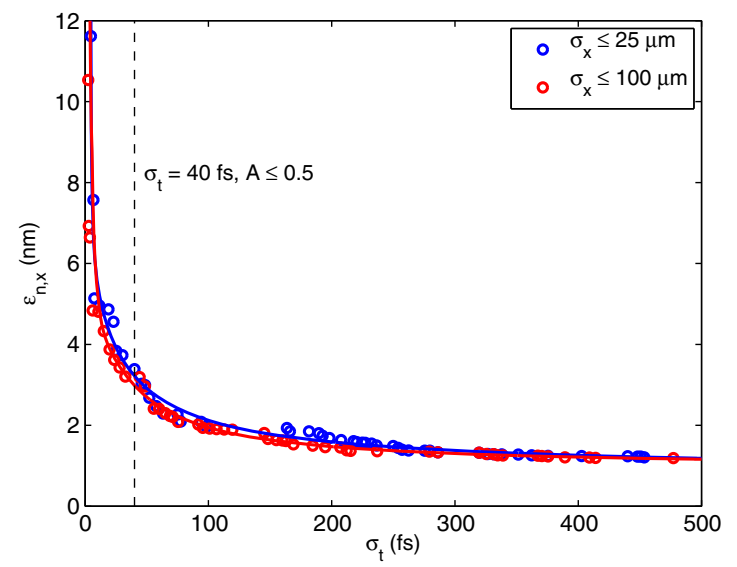

FIG. 3. The optimal emittances as a function of bunch length for a final beam size of $\sigma_{x} \leq 25 \mu \mathrm{m}$ (blue) and $\sigma_{x} \leq 100 \mu \mathrm{m}$ (red), respectively.

aspect ratio range of roughly 0.006 to 0.1 , verifying the fitted scaling law.

With these results, we fixed the charge to $10^{6}$ electrons and reoptimized, this time minimizing the emittance as a function of bunch length. Figure 3 shows the results for two final beam spot sizes at the sample: $\sigma_{x} \leq 25$ and $100 \mu \mathrm{m}$ and demonstrates that the emittance scales weakly with spot size for final beam sizes of $\sigma_{x} \geq 25 \mu \mathrm{m}$. Additionally, the data shows the emittance scales weakly with the final bunch length down to roughly $\sigma_{t} \sim 100$ fs. For shorter bunch lengths the emittance suffers, though we note the existence of feasible solutions with small emittances $\epsilon_{n, x} \lesssim 5 \mathrm{~nm}$ for bunch lengths as short as $\sigma_{t} \sim 5 \mathrm{fs}$. Note, for this plot and all similar ones, we fit a rational polynomial to the Pareto front in order to better guide the eye and to aid estimating and interpolating between points on the front. The dashed black line in this plot indicates where the optimizer selected initial rms laser sizes corresponding to an initial beam aspect ratio of $A \approx 0.5$. Beams to the right of this line are considered to be created in the

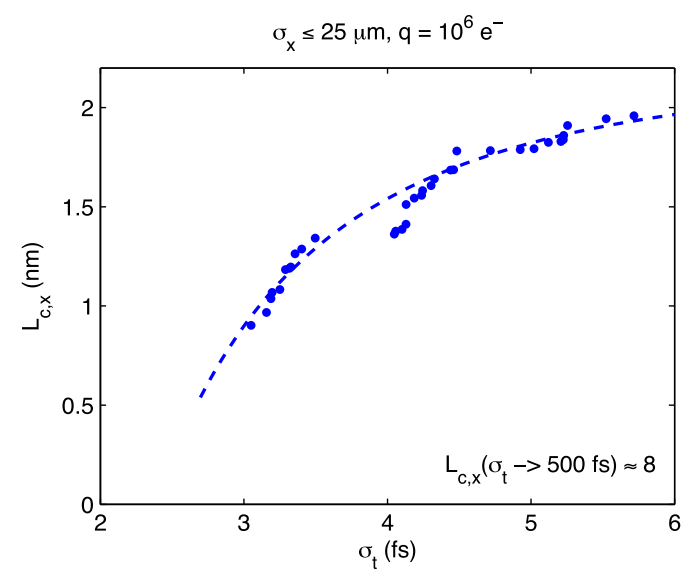

FIG. 4. Optimal coherence length as a function of bunch length at the sample for a final beam spot size of $\sigma_{x} \leq 25 \mu \mathrm{m}$.
TABLE III. Average optimized beam line element positions.

\begin{tabular}{lc}
\hline \hline Element & Position \\
\hline Solenoid 1 & $0.25 \mathrm{~m}$ \\
Buncher cavity & $0.48 \mathrm{~m}$ \\
Solenoid 2 & $1.07 \mathrm{~m}$ \\
Sample pinhole & $2.63 \mathrm{~m}$ \\
\hline \hline
\end{tabular}

long pulse regime. It is instructive to also estimate the disordered induced heating (DIH) of the beam for this data. To do so we assume a uniform beam with equivalent rms sizes. From this, the volume of the beam after being emitted from the cathode follows:

$$
\begin{aligned}
V & =\pi R^{2} L \approx \pi\left(2 \sigma_{x}\right)^{2} \cdot \frac{1}{2} \frac{e E_{0}}{m}\left(\sqrt{12} \sigma_{t}\right)^{2} \\
& \approx \frac{24 \pi E_{0}}{m c^{2}[\mathrm{eV}]} \sigma_{x}^{2}\left(c \sigma_{t}\right)^{2} .
\end{aligned}
$$

From this we estimate the effect of DIH using the formula given by Maxson: $\Delta k T[\mathrm{eV}]=1.04 \times 10^{-9}\left(n_{0}\left[\mathrm{~m}^{-3}\right]\right)^{1 / 3}$ [30], where $n_{0}$ is the electron number density. For the shortest final bunch lengths, the DIH estimate is roughly $150 \mathrm{meV}$. Since this heating occurs near the cathode, this would correspond to roughly a factor of $\sqrt{150 / 35} \approx 2$ increase in the emittance, and would subsequently reduce the coherence length by a factor of 2 . It is important to note that this estimate may indicate that DIH will be important, however, the true estimate of the effect depends on the detailed dynamics of the initial expansion of the beam, and lies beyond the scope of this work.

\section{B. Optimal coherence length}

Using the emittance vs bunch length solutions for a final spot size of $25 \mu \mathrm{m}$ in Fig. 3 as a seed, optimizations for the maximum transverse coherence length were performed.

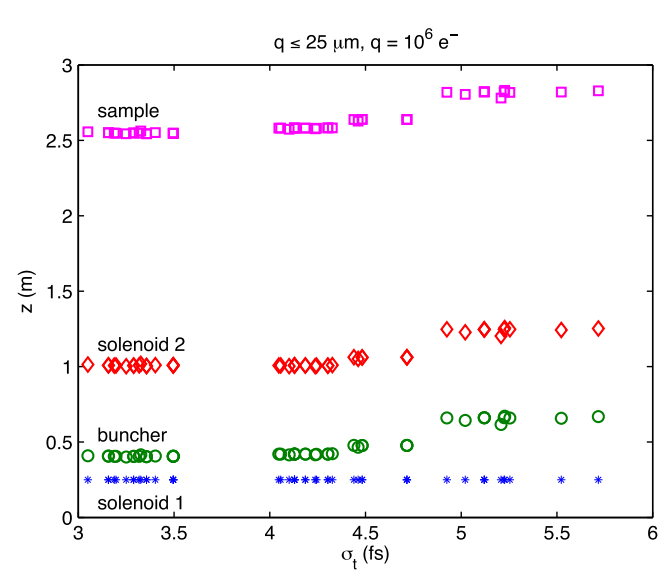

FIG. 5. Optimal beam line element positions for a final beam spot size of $\sigma_{z} \leq 25 \mu \mathrm{m}$. 


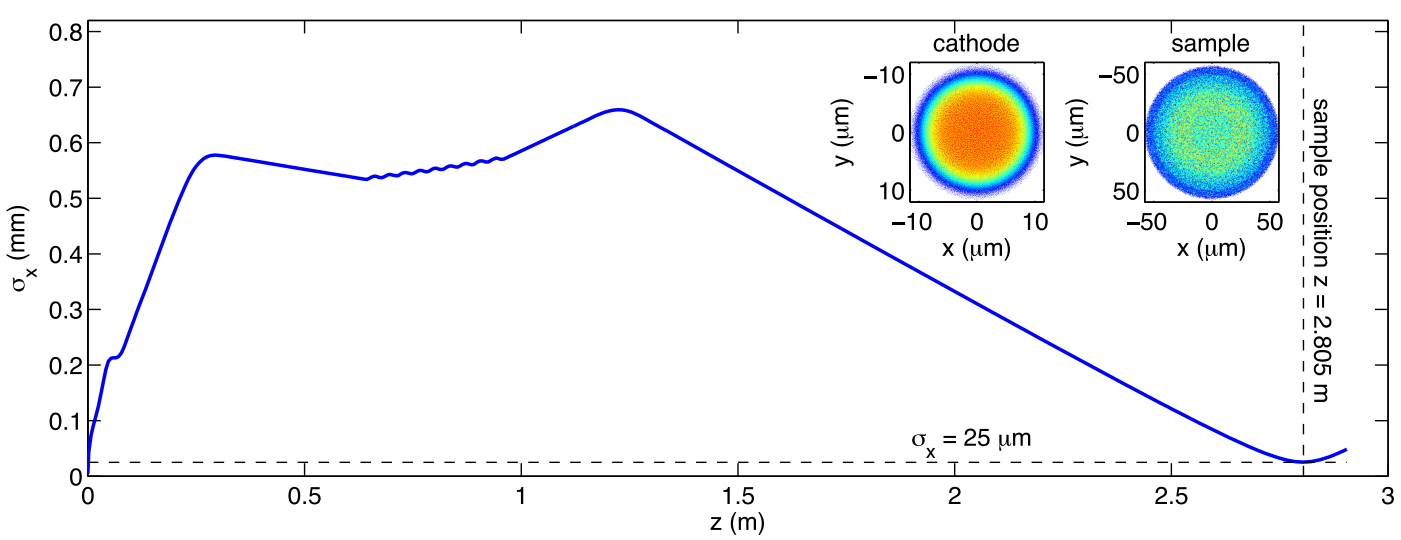

(a)

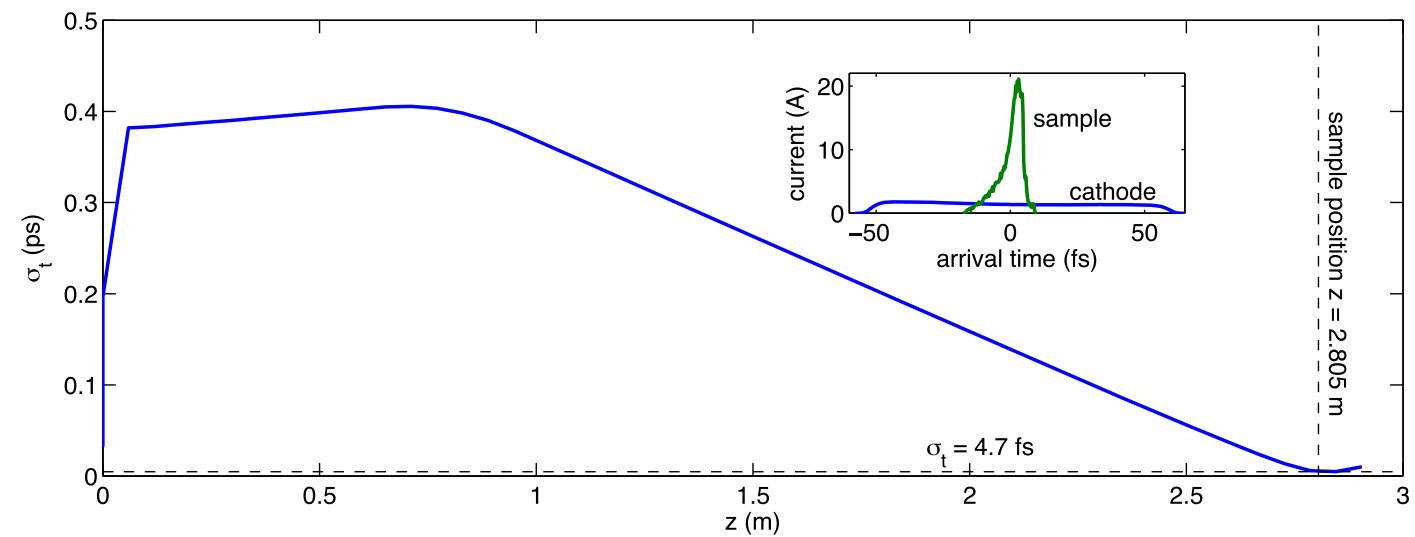

(b)

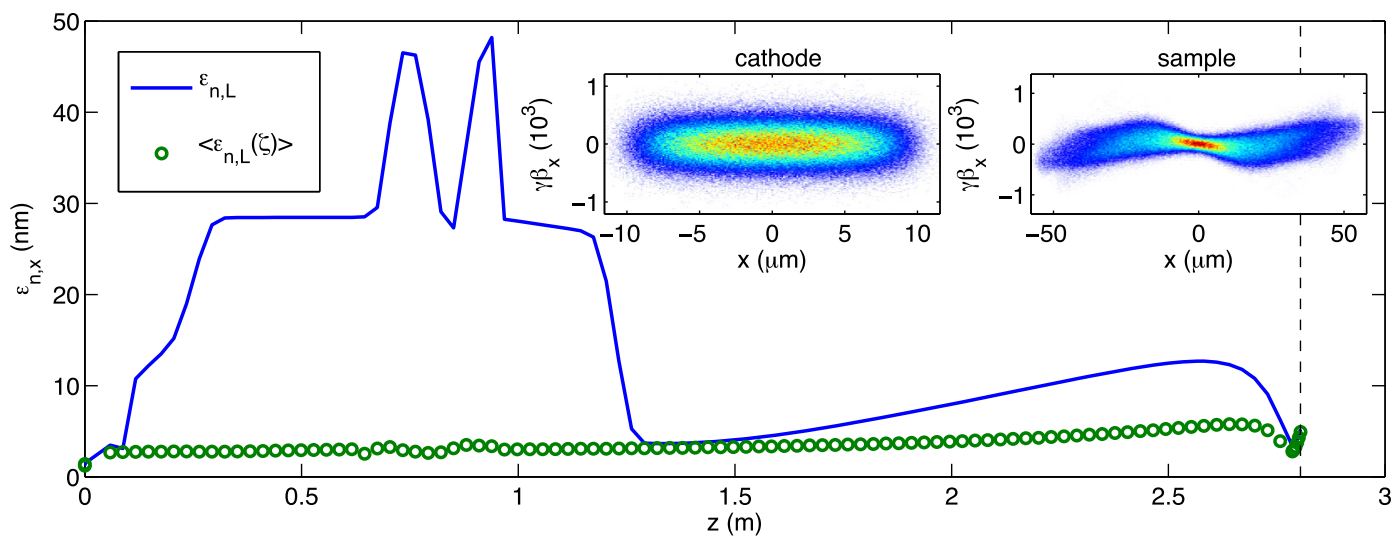

(c)

FIG. 6. Transverse (a) and longitudinal (b) rms beam size along the beam line, as well as the transverse emittance (c). Insets show the transverse (a) and longitudinal (b) beam distributions and (c) transverse phase spaces at the cathode and sample locations.

Figure 4 shows the optimal coherence length as a function of the final bunch length $\sigma_{t}$, constrained so that $\sigma_{x} \leq 25 \mu \mathrm{m}$. In particular, the data shows a relative coherence length of $L_{c, x} / \sigma_{x} \approx 0.07 \mathrm{~nm} / \mu \mathrm{m}$ for a final bunch length of $\sigma_{t} \approx 5$ fs. While the optimizer did not choose solutions at longer bunch lengths in this optimization, using the emittance data in Fig. 3 and the expression for the coherence length at beam waist, $L_{c, x}=\chi \sigma_{x} / \epsilon_{n, x}$, we estimate the relative coherence length at 30,100 , and $500 \mathrm{fs}$ to be $0.1,0.2$, and $0.3 \mathrm{~nm} / \mu \mathrm{m}$, respectively. This implies $L_{c, x}\left(\sigma_{t} \rightarrow 500 \mathrm{fs}\right) \rightarrow 8 \mathrm{~nm}$, as displayed in the figure. It is instructive to compare these results to those found in [26]. At roughly the same bunch length $(5 \mathrm{fs})$, the relative coherence from $\mathrm{Han}$ is about $0.014 \mathrm{~nm} / \mu \mathrm{m}$, implying a factor of 5 improvement in our results. We note, however, that an MTE of $600 \mathrm{meV}$ and a max 
accelerating field of $80 \mathrm{MV} / \mathrm{m}$ were used in [26]. Accounting for these differences would bring the relative coherence length in [26] up to 0.07, in agreement with this work. Thus, we point out the majority of the improvement comes from using a better cathode MTE.

In addition to determining the optimal coherence length, the optimizations producing the data in Fig. 4 also provide information about the optimal positioning of the beam line elements in each setup. Table III displays the element positions averaged over the optimization data shown in Fig. 5.

\section{Example solution}

In order to get a better feel of the beam dynamics determined by the coherence length optimizations, we ran a single example solution from the coherence vs final bunch length fronts shown in Fig. 4. This solution has a kinetic energy of $4.5 \mathrm{MeV}$, typical of all of the simulations presented in this work. Table IV displays the resulting relevant beam parameters. Of note are the initial laser sizes of $\sigma_{x, i} \approx 5 \mu \mathrm{m}$ and $\sigma_{t, i} \approx 32 \mathrm{fs}$. These correspond to an initial beam aspect ratio of roughly $4 \times 10^{4}$. We note that though the optimizer did not generate solutions here at longer bunch lengths, the optimal emittance results suggest viable solutions for longer bunches do exist. Figure 6(a) shows the transverse rms beam size along the beam line, as well as the initial transverse laser profile and the final electron transverse distribution at the sample. The optimizer chose a roughly flattop transverse laser profile with $\sigma_{x} \approx 5 \mu \mathrm{m}$. Figure 6(b) shows the rms bunch length, and the initial temporal current profile produced by the laser, and the electron beam current profile at the sample. The use of the buncher cavity allows for a fairly constant bunch length along the beam line up to the cavity, where the buncher applies an energy chirp which results in the bunch being compressed by the time it reaches the screen. The relevant emittance data along the beam line is shown in Fig. 6(c). Shown in solid blue is the transverse emittance with the angular velocity due to the solenoid removed.

TABLE IV. Example parameters and results.

\begin{tabular}{lc}
\hline \hline Parameter & Simulated value \\
\hline$q$ & $10^{6}$ electrons \\
Gun phase (from peak field) & $-47 \mathrm{deg}$ \\
Buncher phase (from on-crest) & -90 \\
Buncher peak power & $22.5 \mathrm{MW}$ \\
Laser $\sigma_{x, i}$ & $4.68 \mu \mathrm{m}$ \\
Laser $\sigma_{t, i}$ & $32 \mathrm{fs}$ \\
Aspect ratio A & $4 \times 10^{4}$ \\
KE & $4.5 \mathrm{MeV}$ \\
$\sigma_{x}$ & $\leq 25 \mu \mathrm{m}$ \\
$\sigma_{t}$ & $5 \mathrm{fs}$ \\
$\epsilon_{n, x}$ & $5.2 \mathrm{~nm}$ \\
$L_{c, x}$ & $1.85 \mathrm{~nm}$ \\
\hline \hline
\end{tabular}

Shown in green is the slice emittance, computed by averaging over the (30) individual slices. As anticipated, the individual slice phase spaces are nearly aligned at the sample.

\section{CONCLUSION}

In this work, we have presented a multi-MeV NCRF gun based single-shot UED layout determined by MOGA optimization of space charge simulations. For a long final bunch of $500 \mathrm{fs}$ or less, the emittance for this setup scales as $q^{2 / 3}$, in agreement with the predictions for a long initial beam aspect ratio $[24,25]$. Emittances as low as $2-5 \mathrm{~nm}$ were found for final bunch lengths ranging from roughly $500 \mathrm{fs}$ down to 5 fs for a bunch charge of $10^{6}$ electrons. In addition to computing the optimal emittances as a function of bunch length, optimizations of the coherence length as a function the final bunch length produced coherence lengths suitable for single-shot UED experiments with a final electron beam spot size of $\sigma_{x} \geq 25$ and bunch charge of $q \leq 10^{6}$ electrons. In particular, direct optimization of the coherence length produced relative coherence lengths as low as $0.07 \mathrm{~nm} / \mu \mathrm{m}$ for a final bunch length of $\sigma_{t} \approx 5$ fs. Estimating the relative coherence length using optimal emittance data for final bunch lengths of 30 and 100 fs yields relative coherence lengths of 0.1 and $0.2 \mathrm{~nm} / \mu \mathrm{m}$, respectively. Based on the $q^{2 / 3}$ scaling demonstrated in the optimal emittance results, we estimate a factor of 4.6 improvement in the relative coherence numbers $(0.3,0.5$, and $0.92 \mathrm{~nm} / \mu$ m respectively) if the bunch charge was reduced to $10^{5}$ electrons.

\section{ACKNOWLEDGMENTS}

This paper was supported by the NSF, Award No. PHY 1416318.

[1] C. Davisson and L. H. Germer, Diffraction of Electrons by a Crystal of Nickel, Phys. Rev. 30, 705 (1927).

[2] J. C. Williamson, J. Cao, H. Ihee, H. Frey, and A. H. Zewail, Clocking transient chemical changes by ultrafast electron diffraction, Nature (London) 386, 159 (1997).

[3] H. Ihee, V. A. Lobastov, U. M. Gomez, B. M. Goodson, R. Srinivasan, C.-Y. Ruan, and A. H. Zewail, Direct Imaging of Transient Molecular Structures with Ultrafast Diffraction, Science 291, 458 (2001).

[4] B. J. Siwick, J. R. Dwyer, R. E. Jordan, and R. J. D. Miller, Ultrafast electron optics: Propagation dynamics of femtosecond electron packets, J. Appl. Phys. 92, 1643 (2002).

[5] B. J. Siwick, J. R. Dwyer, R. E. Jordan, and R. J. D. Miller, An Atomic-Level View of Melting Using Femtosecond Electron Diffraction, Science 302, 1382 (2003).

[6] M. Harb, R. Ernstorfer, C. T. Hebeisen, G. Sciaini, W. Peng, T. Dartigalongue, M. A. Eriksson, M. G. Lagally, S. G. Kruglik, and R. J. D. Miller, Electronically Driven Structure Changes of Si Captured by Femtosecond Electron Diffraction, Phys. Rev. Lett. 100, 155504 (2008). 
[7] G. Sciaini, M. Harb, S. G. Kruglik, T. Payer, C. T. Hebeisen, F.-J. M. z. Heringdorf, M. Yamaguchi, M. H.-v. Hoegen, R. Ernstorfer, and R. J.D. Miller, Electronic acceleration of atomic motions and disordering in bismuth, Nature (London) 458, 56 (2009).

[8] G. Sciaini and R. J.D. Miller, Femtosecond electron diffraction: heralding the era of atomically resolved dynamics, Rep. Prog. Phys. 74, 096101 (2011).

[9] S. Lahme, C. Kealhofer, F. Krausz, and P. Baum, Femtosecond single-electron diffraction, Struct. Dyn. 1, 034303 (2014).

[10] B. Siwick, J. Dwyer, R. Jordan, and R. Miller, Femtosecond electron diffraction studies of strongly driven structural phase transitions, Chem. Phys. 299, 285 (2004).

[11] T. van Oudheusden, E. F. de Jong, S. B. van der Geer, W. P. E. M. O. 't Root, O. J. Luiten, and B. J. Siwick, Electron source concept for single-shot sub-100 fs electron diffraction in the $100 \mathrm{keV}$ range, J. Appl. Phys. 102, 093501 (2007).

[12] M. Harb, Investigating photoinduced structural changes in Si using femtosecond electron diffraction, Ph.D. thesis, University of Toronto, 2009.

[13] T. van Oudheusden, P. L. E. M. Pasmans, S. B. van der Geer, M. J. de Loos, M. J. van der Wiel, and O. J. Luiten, Compression of Subrelativistic Space-Charge-Dominated Electron Bunches for Single-Shot Femtosecond Electron Diffraction, Phys. Rev. Lett. 105, 264801 (2010).

[14] R. P. Chatelain, V. R. Morrison, C. Godbout, and B. J. Siwick, Ultrafast electron diffraction with radio-frequency compressed electron pulses, Appl. Phys. Lett. 101, 081901 (2012).

[15] M. Gao, C. Lu, H. Jean-Ruel, L. C. Liu, A. Marx, K. Onda, S.-y. Koshihara, Y. Nakano, X. Shao, T. Hiramatsu, G. Saito, H. Yamochi, R. R. Cooney, G. Moriena, G. Sciaini, and R. J. D. Miller, Mapping molecular motions leading to charge delocalization with ultrabright electrons, Nature (London) 496, 343 (2013).

[16] W. J. Engelen, M. A. van der Heijden, D. J. Bakker, E. J. D. Vredenbregt, and O.J. Luiten, High-coherence electron bunches produced by femtosecond photoionization, Nat. Commun. 4, 1693 (2013).

[17] A. J. McCulloch, D. V. Sheludko, M. Junker, and R. E. Scholten, High-coherence picosecond electron bunches from cold atoms, Nat. Commun. 4, 1692 (2013).

[18] P. Musumeci, J. T. Moody, R. J. England, J. B. Rosenzweig, and T. Tran, Experimental Generation and Characterization of Uniformly Filled Ellipsoidal Electron-Beam Distributions, Phys. Rev. Lett. 100, 244801 (2008).

[19] C. Gulliford, A. Bartnik, I. Bazarov, L. Cultrera, J. Dobbins, B. Dunham, F. Gonzalez, S. Karkare, H. Lee, H. Li, Y. Li, X. Liu, J. Maxson, C. Nguyen, K. Smolenski, and Z. Zhao, Demonstration of low emittance in the Cornell energy recovery linac injector prototype, Phys. Rev. ST Accel. Beams 16, 073401 (2013).

[20] C. Gulliford, A. Bartnik, I. Bazarov, B. Dunham, and L. Cultrera, Demonstration of cathode emittance dominated high bunch charge beams in a DC gun-based photoinjector, Appl. Phys. Lett. 106, 094101 (2015).

[21] A. Bartnik, C. Gulliford, I. Bazarov, L. Cultera, and B. Dunham, Operational experience with nanocoulomb bunch charges in the Cornell photoinjector, Phys. Rev. ST Accel. Beams 18, 083401 (2015).

[22] C. Gulliford, A. Bartnik, and I. Bazarov, Multiobjective optimizations of a novel cryocooled dc gun based ultrafast electron diffraction beam line, Phys. Rev. Accel. Beams 19, 093402 (2016).

[23] J. Maxson, L. Cultrera, C. Gulliford, and I. Bazarov, Measurement of the tradeoff between intrinsic emittance and quantum efficiency from a NaKSb photocathode near threshold, Appl. Phys. Lett. 106, 234102 (2015).

[24] R. K. Li, K. G. Roberts, C. M. Scoby, H. To, and P. Musumeci, Nanometer emittance ultralow charge beams from rf photoinjectors, Phys. Rev. ST Accel. Beams 15, 090702 (2012).

[25] D. Filippetto, P. Musumeci, M. Zolotorev, and G. Stupakov, Maximum current density and beam brightness achievable by laser-driven electron sources, Phys. Rev. ST Accel. Beams 17, 024201 (2014).

[26] J.-H. Han, Production of a sub-10 fs electron beam with $10^{7}$ electrons, Phys. Rev. ST Accel. Beams 14, 050101 (2011).

[27] R. Neal, The Stanford Two-Mile Accelerator (W.A. Benjamin, New York, 1968), Vol. 1.

[28] http://laacg.lanl.gov/laacg/services/download_sf.phtml.

[29] I. V. Bazarov, A. Kim, M.N. Lakshmanan, and J. M. Maxson, Comparison of dc and superconducting rf photoemission guns for high brightness high average current beam production, Phys. Rev. ST Accel. Beams 14, 072001 (2011).

[30] J. M. Maxson, I. V. Bazarov, W. Wan, H. A. Padmore, and C. E. Coleman-Smith, Fundamental photoemission brightness limit from disorder induced heating, New J. Phys. 15, 103024 (2013). 